\title{
A Clinico- Haematological Profile of Dengue Fever among Pediatric Patients in a North Indian Tertiary Hospital
}

\author{
Mahima Bansal ${ }^{1}$, Manish Agrawal ${ }^{2}$ \\ ${ }^{1}$ Post Graduate Student, Department of Pediatrics, Saraswathi Institute of Medical Sciences, Hapur, Uttar Pradesh, India, ${ }^{2}$ Professor and Head, Department of Pediatrics, \\ Saraswathi Institute of Medical Sciences, Hapur, Uttar Pradesh, India.
}

\section{Abstract}

Background: Over the years, Dengue fever outbreaks are increasing both in number and distribution in India. Subjects and Methods: In this study all probable cases of dengue fever in age group 2-14 years were included in study. Their detailed clinical and laboratory profile were recorded in pre designed proforma. Cases were classified according to WHO 2009 classification. Patients with positive for Dengue markers (IgM/NS1 antigen) are considered as Dengue fever. Results: Total of 50 cases were included in the study out of these $56 \%$ were males and $44 \%$ were females with majority of them were in 6-14 years of age. Majority of patients presented with headache (88\%) followed by muscular pain $(82 \%)$. Hess test, positive in $(24 \%)$, with Spleenomegaly in $16 \%$ and Hepatomegaly in $14 \%$. Lab parameters revealed thrombocytopenia and leucopenia. Only $2 \%$ patient expired with $88 \%$ were discharged with improvement. A total of $38 \%$ subjects had dengue like fever, $26 \%$ subjects presented with dengue, $34 \%$ had dengue with warning signs and $2 \%$ subjects had severe dengue. Conclusion: Dengue fever can presents with varied clinical and laboratory manifestation. Early diagnosis and appropriate management can reduce morbidity and mortality markedly.

Keywords: Dengue, Fever, Pediatric patients.

Corresponding Author: Dr. Manish Agrawal, Professor and Head, Department of Pediatrics, Saraswathi Institute of Medical Sciences, Hapur, Uttar Pradesh, India.

Received: March 2019

Accepted: March 2019

\section{Introduction}

Dengue is an acute viral infection with potential fatal complications. ${ }^{[1]}$ According to the World Health Organization, the incidence of dengue globally has shot up 30 -fold in the past 50 years. The cumulative dengue diseases burden has attained an unprecedented proportion in recent times with a sharp increase in the size of human population at risk. Dengue disease presents highly complex pathophysiological, economic, and ecologic problems. ${ }^{[2]}$

A recent study done at the University of Oxford using a map-based approach to model how many dengue cases were occurring in various parts of the world, estimated that India had the largest number of dengue cases, with about 33 million apparent and another 100 million asymptomatic infections occurring annually. ${ }^{[3]}$ Dengue viruses (DV) belong to family Flaviviridae and there are four serotypes of the virus referred to as DV-1, DV-2, DV-3 and DV-4.It is transmitted mainly by Aedes aegypti mosquito. All four serotypes can cause the full spectrum of disease from a subclinical infection to a mild self limiting disease, the dengue fever (DF) and a severe disease, the dengue haemorrhagic fever/dengue shock syndrome (DHF/ DSS) that may be fatal. ${ }^{[4]}$
Due to limitations of World Health Organisation (WHO) 1997 dengue classification guidelines, WHO guidelines were revised in $2009,{ }^{[5]}$ as dengue without warning signs, dengue with warning signs and severe dengue. ${ }^{[6-8]}$ Potential variability in clinical picture of dengue disease and impact of heterogeneous genetic and geographical factors towards this spectrum summons for extensive studies of clinical picture and prognosis in dengue disease in different geographical location. Lack of such data from North India lead us to undertake this study.

This work will clarify in detail the incidence of dengue fever in dengue like fever, clinical picture and laboratory parameters of serologically confirmed hospitalised cases of dengue fever.

\section{Subjects and Methods}

The present study was an observational study conducted in Saraswathi Institute of Medical Sciences. A total of 50 patients were recruited in the study. In the present research, all cases of Dengue like fever were considered in the study and out of these patients with positive for Dengue IgM/NS1 antigen were considered Dengue positive patients.

Inclusion Criteria: 
All probable cases of Dengue fever ranging from 02-14 years of age

\section{Exclusion Criteria:}

Cases presented with fever and other symptoms like UTI Pneumonia, Malaria, Typhoid, ASOM

Ethical Approval was taken from the Institutional Ethical Committee after explaining the aim and objectives of the study. A written informed consent was taken prior to the investigation after detailed information given to the participants regarding the study. Fever among patients was screened for Dengue through a thorough history, detailed examination and lab investigations, cases were admitted, treated and followed up for the treatment outcomes.

\section{Statistical Analysis:}

Statistical analysis was performed using SPSS (Statistical Package for the Social Sciences) for Windows (version 15.0). Categorical variables were described as frequency (percentage), mean \pm standard deviation was used for continuous parameters. Differences between two groups were compared by the Student $\mathrm{T}$ test. For non-parametric variables, the data are presented as median (min-max). In this case, the nonparametric Mann-Whitney test was used for statistical comparisons. Categorical variables were compared between two or more groups using the Chi-square test. For all analyses, a two-tailed p-value of $<0.05$ was considered statistically significant.

\section{Results}

The age wise distribution of patients showed that majority of them was in the age group of 6-14 years. The mean age of the patients was $9.85+3.34$ years (mean+s.d.) and range $=3$ to 14 years. A majority were males $(56 \%)$ and rest were females $(44 \%)$. The highest proportion was of school children ( $>6$ years) and more were males [Table 1].

The incidence of dengue was seen among 31 patients in the study sample. All subjects presented with fever (100\%). A majority of study subjects (88\%) presented with headache followed by muscular pain $(82 \%)$. Nausea and vomiting seen in $(80 \%)$ of study subjects followed by $74 \%$ subjects presenting with retro-orbital pain and 54\% with abdominal pain.

Regarding bleeding manifestations, on performing Hess test, it was positive in 12 patients (24\%) with petechial rash in
6 patients (12\%), mucosal bleed in 4 patients $(8 \%)$, ecchymotic patch in 3 patients $(6 \%)$ and only 1 patient (2\%) had a dark color stool. Also, $14 \%$ \& $16 \%$ patients had hepatomegaly and splenomegaly repectively.

All vital parameters of the study participants as per age categories such as mean Systolic BP, mean Diastolic BP, Pulse and Weight were significantly higher among school age children ( $>6$ years) as compared to pre-school and toddler age children $(\mathrm{p}<0.05)$.

Thrombocytopenia comparison with age group and symptoms showed that a greater proportion of school age children ( $>6$ years) were seen to have low platelet count, however the difference was statistically not significant [Table 2]. Also, it was noticed that subjects who had platelet count upto 50,000 had a significantly higher proportion of positive Hess test $(\mathrm{p}<0.05)$ and ecchymotic rash $(\mathrm{p}<0.05)$. The remaining symptoms were not significantly associated with platelet count [Table 3].

When markers for Dengue were assessed among study patients, it was seen that $\mathrm{n} 44 \%$ patients had an abnormal NS1 value. Only $6 \%$ patients had just abnormal IgM values and none of the patient had abnormal IgG values individually. A combined abnormal NS1 and IgM values were seen in 6 patients a combined abnormal NS1 and IgG values were seen in 5 patients, and a combined abnormal $\operatorname{IgG}$ and $\operatorname{IgM}$ values were seen in 9 patients. Nearly $70 \%$ patients had at least one abnormal dengue biomarker [Table 4].

The overall diagnosis revealed that a total of $38 \%$ subjects had dengue like fever, $26 \%$ subjects presented with dengue, $34 \%$ had dengue with warning signs and $2 \%$ subjects had severe dengue among study participants. Ultimately, 44 subjects (88\%) were discharged with improvement, 1 patient expired, and 5 patients were LAMA (Leave against Medical Advice) [Figure 1].

Table 1: Age and Gender distribution of study subjects

\begin{tabular}{|l|l|l|l|l|l|}
\hline \multicolumn{2}{|c}{} & & \multicolumn{2}{|l|}{ Gender } & \multirow{2}{*}{ Total } \\
\cline { 3 - 6 } \multicolumn{2}{|c|}{} & & Females & Males & \\
\hline \multirow{3}{*}{ Age Group } & Toddler (1-3) & $\mathrm{N}$ & 1 & 1 & 2 \\
\cline { 3 - 6 } & & $\%$ & $4.5 \%$ & $3.6 \%$ & $4.0 \%$ \\
\cline { 3 - 6 } & Preschool (>3-6) & $\mathrm{N}$ & 5 & 3 & 8 \\
\cline { 3 - 6 } & & $\%$ & $22.7 \%$ & $10.7 \%$ & $16.0 \%$ \\
\cline { 3 - 6 } & \multirow{2}{*}{ School (<6) } & $\mathrm{N}$ & 16 & 24 & 40 \\
\cline { 3 - 6 } & & $\%$ & $72.7 \%$ & $85.7 \%$ & $80.0 \%$ \\
\hline Total & $\mathrm{N}$ & 22 & 28 & 50 \\
\hline
\end{tabular}

Table 2: Thrombocytopenia vs age group

\begin{tabular}{|c|c|c|c|c|c|c|c|}
\hline \multicolumn{2}{|l|}{ Age category } & \multicolumn{5}{|c|}{ Platelets } & \multirow[t]{2}{*}{ Total } \\
\hline & & $<20 \mathrm{~K}$ & $20 \mathrm{~K}-50 \mathrm{~K}$ & $50 \mathrm{~K}-100 \mathrm{~K}$ & 100K-150K & $>150 \mathrm{~K}$ & \\
\hline \multirow[t]{2}{*}{ Toddler (1-3) } & $\mathrm{N}$ & 0 & 0 & 0 & 0 & 2 & 2 \\
\hline & $\%$ & $0.0 \%$ & $0.0 \%$ & $0.0 \%$ & $0.0 \%$ & $18.2 \%$ & $4.0 \%$ \\
\hline \multirow[t]{2}{*}{ Preschool (>3-6) } & $\mathrm{N}$ & 0 & 3 & 3 & 0 & 2 & 8 \\
\hline & $\%$ & $0.0 \%$ & $23.1 \%$ & $21.4 \%$ & $0.0 \%$ & $18.2 \%$ & $16.0 \%$ \\
\hline \multirow[t]{2}{*}{ School $(>6)$} & $\mathrm{N}$ & 3 & 10 & 11 & 9 & 7 & 40 \\
\hline & $\%$ & $100.0 \%$ & $76.9 \%$ & $78.6 \%$ & $100.0 \%$ & $63.6 \%$ & $80.0 \%$ \\
\hline Total & $\mathrm{N}$ & 3 & 13 & 14 & 9 & 11 & 50 \\
\hline
\end{tabular}




\section{Bansal \& Agrawal; Profile of Dengue Fever amang Pediatric Patients}

Table 3: Platelets vs Bleeding manifestations

\begin{tabular}{|c|c|c|c|c|c|c|c|c|}
\hline & & & $<20 K$ & $20 \mathrm{~K}-50 \mathrm{~K}$ & $50 \mathrm{~K}-100 \mathrm{~K}$ & 100K-150K & $>150 \mathrm{~K}$ & p-value \\
\hline \multirow[t]{4}{*}{ Hess test } & \multirow[t]{2}{*}{ No } & $\mathrm{N}$ & 1 & 5 & 13 & 8 & 11 & \multirow[t]{4}{*}{$0.001^{*}$} \\
\hline & & $\%$ & $16.7 \%$ & $45.5 \%$ & $92.9 \%$ & $100.0 \%$ & $100.0 \%$ & \\
\hline & \multirow[t]{2}{*}{ Yes } & $\mathrm{N}$ & 5 & 6 & 1 & 0 & 0 & \\
\hline & & $\%$ & $83.3 \%$ & $54.5 \%$ & $7.1 \%$ & $0.0 \%$ & $0.0 \%$ & \\
\hline \multirow[t]{4}{*}{ Petechial Rash } & \multirow[t]{2}{*}{ No } & $\mathrm{N}$ & 4 & 9 & 13 & 7 & 11 & \multirow[t]{4}{*}{0.309} \\
\hline & & $\%$ & $66.7 \%$ & $81.8 \%$ & $92.9 \%$ & $87.5 \%$ & $100.0 \%$ & \\
\hline & \multirow[t]{2}{*}{ Yes } & $\mathrm{N}$ & 2 & 2 & 1 & 1 & 0 & \\
\hline & & $\%$ & $33.3 \%$ & $18.2 \%$ & $7.1 \%$ & $12.5 \%$ & $0.0 \%$ & \\
\hline \multirow[t]{4}{*}{ Ecchymotic Patch } & \multirow[t]{2}{*}{ No } & $\mathrm{N}$ & 4 & 10 & 14 & 8 & 11 & \multirow[t]{4}{*}{$0.037 *$} \\
\hline & & $\%$ & $66.7 \%$ & $90.9 \%$ & $100.0 \%$ & $100.0 \%$ & $100.0 \%$ & \\
\hline & \multirow[t]{2}{*}{ Yes } & $\mathrm{N}$ & 2 & 1 & 0 & 0 & 0 & \\
\hline & & $\%$ & $33.3 \%$ & $9.1 \%$ & $0.0 \%$ & $0.0 \%$ & $0.0 \%$ & \\
\hline \multirow[t]{4}{*}{ Mucosal Bleed } & \multirow[t]{2}{*}{ No } & $\mathrm{N}$ & 5 & 10 & 12 & 8 & 11 & \multirow[t]{4}{*}{0.552} \\
\hline & & $\%$ & $83.3 \%$ & $90.9 \%$ & $85.7 \%$ & $100.0 \%$ & $100.0 \%$ & \\
\hline & \multirow[t]{2}{*}{ Yes } & $\mathrm{N}$ & 1 & 1 & 2 & 0 & 0 & \\
\hline & & $\%$ & $16.7 \%$ & $9.1 \%$ & $14.3 \%$ & $0.0 \%$ & $0.0 \%$ & \\
\hline \multirow[t]{4}{*}{ Dark Stools } & \multirow[t]{2}{*}{ No } & $\mathrm{N}$ & 6 & 10 & 13 & 8 & 11 & \multirow[t]{4}{*}{0.709} \\
\hline & & $\%$ & $100.0 \%$ & $90.9 \%$ & $92.9 \%$ & $100.0 \%$ & $100.0 \%$ & \\
\hline & \multirow[t]{2}{*}{ Yes } & $\mathrm{N}$ & 0 & 1 & 0 & 0 & 0 & \\
\hline & & $\%$ & $0.0 \%$ & $9.1 \%$ & $0.0 \%$ & $0.0 \%$ & $0.0 \%$ & \\
\hline
\end{tabular}

\begin{tabular}{|c|c|c|c|c|}
\hline \multirow[t]{2}{*}{$\begin{array}{l}\text { Dengue } \\
\text { biomarkers }\end{array}$} & \multirow[t]{2}{*}{ Frequency } & \multirow[t]{2}{*}{$\begin{array}{l}\text { Percent } \\
(\%)\end{array}$} & \multicolumn{2}{|c|}{$\begin{array}{lll}\begin{array}{l}\text { Platelet count } \\
\text { lakhs) }\end{array} & \text { (in } \\
\end{array}$} \\
\hline & & & Mean & SD \\
\hline NS1 only & 22 & 44.0 & 1.47 & 1.26 \\
\hline IgM only & 3 & 6.0 & 0.72 & 0.56 \\
\hline IgG only & 0 & 0.0 & - & - \\
\hline $\mathrm{NS} 1+\mathrm{IgM}$ & 6 & 12.0 & 0.56 & 0.25 \\
\hline $\mathrm{NS} 1+\mathrm{IgG}$ & 5 & 10.0 & 0.58 & 0.27 \\
\hline $\mathrm{IgM}+\mathrm{IgG}$ & 9 & 18.0 & 0.85 & 1.09 \\
\hline Any of the three & 35 & 70.0 & 1.22 & 1.18 \\
\hline
\end{tabular}

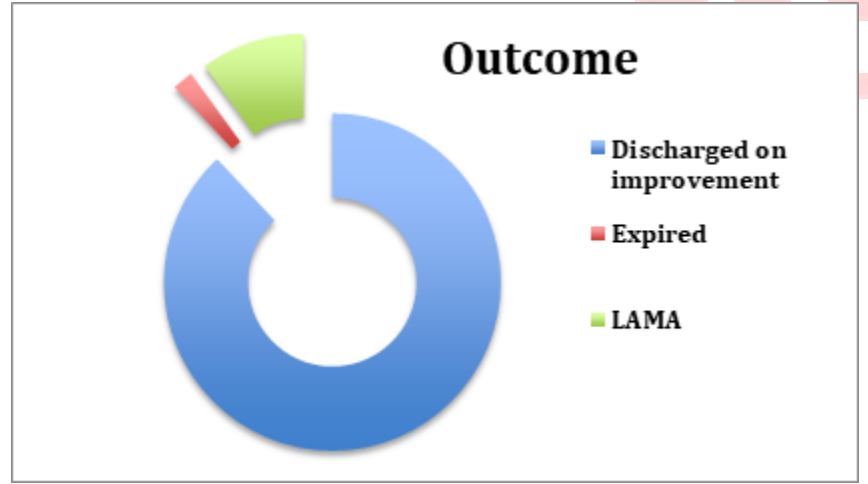

Outcome

Figure 1: Outcome of study subjects

\section{Discussion}

The present study is an attempt to describe the salient clinical as well as laboratory findings of serologically confirmed pediatric hospitalized cases of dengue fever. The age wise distribution of study participants showed that majority of them was in the age group of 6-14 years with mean age of study population being around 10 years. This is commonly affected age group as reported by other studies elsewhere. ${ }^{[9-11]}$ Study done by Mital $\mathrm{H}$ et al had a mean age of 10.3 years, ${ }^{[9]}$ and the study performed by Jain et al had the maximum proportion of children in 5-10 year age group. ${ }^{[10]}$

The gender wise distribution of study participants showed that majority of them were males $(56 \%)$. The finding is comparable to study by Sahana et al in 2010 (reporting 68\% males), Adam et. al, in 2018 (reporting 62\% males), and Jain et al in 2016 (reporting $53 \%$ males). ${ }^{[10-12]}$ This could be attributed to covered dress used by females, which may be the cause for fewer incidences. The other symptoms seen in the present study also goes with previous studies. ${ }^{[13]}$ The study performed by Ahmed $\mathrm{S}$ et al showed similar incidence of common clinical features such as fever (97\%), vomiting $(68 \%)$, abdominal pain $(68 \%)$ and rashes $(65 \%)$.

A platelet count $<50000 / \mathrm{mm} 3$ in dengue has a six-fold higher mortality but in our study only Hess test and petechial rash were seen with low platelet count in $24 \%$ and $12 \%$ patients respectively. Previous studies have reported similar findings, and this finding points towards the fact that bleeding in dengue is multifactorial. The study conducted by Dhooria et al showed that all patients had platelet counts less than 100,000 /cumm, while $59 \%$ of cases had platelet count less than 50,000/cumm. Twelve children had platelet counts below 20,000/cumm but only one child had evidence of spontaneous bleeding. ${ }^{[14]}$ Various factors cause bleeding in dengue including thrombocytopenia, abnormal platelet function, prolongation of prothrombin time, fibrinogen consumption, etc. ${ }^{[15]}$

The most common bleeding manifestations in both severe and nonsevere dengue were petechiae, purpura, and ecchymosis. On performing Hess test, it was seen that a total of 12 individuals (24\%) had a positive Hess test. Low proportion of positive tourniquet test in Indian studies may be due to the darker skin colour in Indian children. ${ }^{[16,17]}$ The study conducted by Mishra et al showed the very few children had a positive tourniquet test. ${ }^{[17]}$ There were a low 
proportion of children with evidence of haemoconcentration in our study group. The percentage increase in haematocrit is an accurate indicator of vascular permeability and plasma leakage.

There was less mortality in the present study group, whereas mortality rate was high in earlier previous studies. This could be due to delay in recognition of epidemic in previous years or delay in seeking medical attention. Case fatality rate (CFR) of the SEAR countries in 2006 was less than $1 \%$. India, Indonesia, Bhutan, and Nepal still have case fatality rates above $1 \% .^{[17]}$ Early diagnosis and improved case management of dengue fever are required to bring down CFR to below $1 \%$.

In our study, Non-Structural protein 1 antigen was positive in $56 \%$ of cases and IgM and IgG was positive in $26 \%$ and $18 \%$ cases. This is less than reported by Jain et al in Rajasthan. ${ }^{[12]}$ In our study all non-severe dengue cases recovered. Among the severe dengue cases 4 cases recovered and 1 child expired due to intractable shock.

The current study showed $44 \%$ prevalence of NS1 antigen only, followed by $6 \% \operatorname{IgM}$ antibody. Another study showed a similar finding, wherein parameters NS1 (33.8\%) and IgM $(48 \%)$ were seen in the study population. The combination, however, was less frequent. ${ }^{[18]}$ The gender and age group comparison of such findings revealed no significant relationship. However, we could not find any study relating to these results. Further exploration is therefore needed.

The present study showed the maximum prevalence of Dengue like fever (38\% subjects), followed by Dengue (26\% subjects) and Dengue with warning signs (34\% subjects). This classification was rendered by WHO in 2009. [19] Similar findings were seen in the study done by Jain el at where dengue without warning signs was seen in $23 \%$ subjects, ${ }^{[12]}$ dengue with warning signs was observed in $65 \%$ subjects, and severe dengue was seen in $12 \%$ subjects.

\section{Conclusion}

The current study was an observational hospital based study. All probable cases of Dengue fever admitted in the hospital were recruited for the study and patients positive for Dengue markers would be considered as dengue fever, while those that were not positive for the three assays were considered dengue negative. All subjects presented with fever. A majority of study subjects presented with headache and muscular pain. A very less percentage of patients had a positive Hess test, followed by an even less frequency of positive Petechial rash, Ecchymotic Patch, and Mucosal bleed. There was correlation between platelet counts and bleeding manifestations with increased frequency of petechial rash, ecchymotic patch, mucosal bleed in patients with thrombocytopenia. A greater proportion of school age children (>6 years) were seen to have lower platelet count, however the difference was statistically not significant. Nearly $70 \%$ subjects had at least one abnormal dengue biomarker, and majority of patients were discharged after improvement.

\section{References}

1. Whitehorn J, Farrar J. Dengue. Br Med Bull 2010; 95 : 161- 73.

2. WHO. Dengue: Guidelines for diagnosis, treatment, prevention, and control in sub-Saharan Africa and 13 countries in South America. Geneva: World Health Organization; 2009

3. Ramaiah R, Jayarama S. Awareness and practices related to dengue fever among rural high school students: a cross sectional study. Int $\mathrm{J}$ Community Med Public Health 2018; 5: 1402-6.

4. WHO. Dengue hemorrhagic fever: Diagnosis, treatment, prevention, and control. Geneva: WHO press;2009:3-106

5. Alexander N, Balmaseda A, Coelho IC, Dimaano E, Hien TT, Hung NT. Multicentre prospective study on dengue classification in four South-east Asian and three Latin American countries. Trop Med Int Health. 2011;16:936-48.

6. WHO: Dengue Guildelines for Diagnosis, Prevention and Control New edition. Geneva, Switzerland: World Health Organisation. 2009.

7. WHO: Handbook for Clinical Management of Dengue. 2012.

8. Barniol J, Gaczkowski R, Barbato EV, da Cunha RV, Salgado D, Martínez E. Usefulness and applicability of the revised dengue case classification by disease: multi-centre study in 18 countries. BMC Infect Dis. 2011;11:106.

9. Mittal H, Faridi MM, Arora SK, Patil R. Clinicohematological profile and platelet trends in children with dengue during 2010 epidemic in north India. Indian J Pediatr. 2012;79:467-71.

10. Sahana KS, Sujatha R. Clinical profile of dengue among children according to revised WHO classification: analysis of a 2012 outbreak from Southern India. Indian J Pediatr 2015;82:109-13

11. Adam AS, Pasaribu S, Wijaya H, Pasaribu AP. Clinical profile and warning sign finding in children with severe dengue and non-severe dengue. IOP Conf. Series: Earth and Environmental Science 125 (2018) 012038

12. Jain H. Clinical profile and outcome of dengue fever in hospitalized children of South Rajasthan, India. Int J ContempPediatr 2016;3:5469.

13. Ahmed S., Arif F., Yahya Y., et al. Dengue fever outbreak in Karachi 2006-a study of profile and outcome of children under 15 years of age. Journal of the Pakistan Medical Association. 2008;58(1):4-8.

14. Dhooria GS, Bhat D, Bains HS. Clinical profile and outcome in children of dengue haemorrhagic fever in North India. Iran J Pediatr 2008; 18:222-228.

15. Krishnamurti C, Kalayanarooj S, Cutting MA, et al. Mechanisms of hemorrhage in dengue without circulatory collapse. Am J Trop Med Hyg 2001; 65:840-847.

16. Kalayanarooj S., Vaughn D. W., Nimmannitya S., et al. Early clinical and laboratory indicators of acute dengue illness. Journal of Infectious Diseases. 1997;176(2):313-321. doi: 10.1086/514047.

17. Mishra S, Ramanathan R, Agarwalla SK. Clinical Profile of Dengue Fever in Children: A Study from Southern Odisha, India. Scientifica Volume 2016, Article ID 6391594, 6 pages. http://dx.doi.org/10.1155/2016/6391594

18. Ravindra M, Nisha KC, Gadgil SA, et al. Association of IgG, IgM antibodies, NS1 antigen and platelet count in the diagnosis of dengue virus infection in patients attending bharatividyapeeth deemed university medical college and hospital, Sangli. International Journal of Contemporary Medical Research 2016;3(10):2942-2943

19. Bandyopadhyay S, Lum LC, Kroeger A. Classifying dengue: a review of the difficulties in using the WHO case classification for dengue haemorrhagic fever. Trop Med Int Health. 2006;11:1238-55. 
Copyright: () the author(s), 2019. It is an open-access article distributed under the terms of the Creative Commons Attribution License (CC BY 4.0), which permits authors to retain ownership of the copyright for their content, and allow anyone to download, reuse, reprint, modify, distribute and/or copy the content as long as the original authors and source are cited.

How to cite this article: Bansal M, Agrawal M. A Clinico- Haematological Profile of Dengue Fever among Pediatric Patients in a North Indian Tertiary Hospital. Asian J. Clin. Pediatr. Neonatol.2019;7(1):55-59.

DOI: dx.doi.org/10.21276/ajcpn.2019.7.1.13

Source of Support: Nil, Conflict of Interest: None declared. 